\title{
TECNOLOGIA DE APLICAÇÃO DE INSETICIDAS NO CONTROLE DA LAGARTA-DO-CARTUCHO NA CULTURA DO MILHO
}

\author{
RAFAEL MARCÃO TAVARES ${ }^{1}$, JOÃO EDUARDO RIBEIRO DA SILVA ${ }^{1}$, \\ GUILHERME SOUSA ALVES ${ }^{1}$, THALES CASSEMIRO ALVES ${ }^{1}$, SÉRGIO MACEDO SILVA ${ }^{1}$ \\ e JOÃO PAULO ARANTES R. CUNHA ${ }^{1}$
}

${ }^{1}$ Universidade Federal de Uberlândia, Instituto de Ciências Agrárias, Caixa Postal 593, 38.400-902, Uberlândia-MG,Brasil; Contatos: rmtagro@gmail.com; johned87@yahoo.com.br; guilhermeagro43@yahoo.com.br; thalescalves@hotmail.com; sergiomacedosilva@yahoo.com.br;jpcunha@iciag.ufu.br

Revista Brasileira de Milho e Sorgo, v.16, n.1, p. 30-42, 2017

\begin{abstract}
RESUMO - A lagarta-do-cartucho (Spodoptera frugiperda) é uma praga-chave na cultura do milho, e aplicações de inseticidas têm sido necessárias para reduzir a população do inseto. O controle desta praga tem sido difícil porque a lagarta encontra-se comumente protegida no interior do cartucho, o que pode reduzir a deposição e eficácia do inseticida. Portanto, este trabalho objetivou avaliar o efeito de taxas de aplicação, pontas de pulverização e inseticidas no controle de $S$. frugiperda no milho. O experimento foi conduzido em delineamento em blocos casualizados, em esquema fatorial $2 \times 2 \times 2+1$ com quatro repetições. Os fatores foram duas taxas de aplicação (100 e $\left.200 \mathrm{~L} \mathrm{ha}^{-1}\right)$, duas pontas de pulverização (jato plano com e sem com indução de ar) e dois inseticidas (triflumuron e fenpropatrina). Também se utilizou um tratamento controle correspondente à aplicação de clorpirifós utilizando a ponta jato plano simples na taxa de $200 \mathrm{~L} \mathrm{ha}^{-1}$. As características avaliadas foram espectro de gotas, deposição de calda, eficácia biológica e produtividade da cultura. A taxa de aplicação de $100 \mathrm{~L} \mathrm{ha}^{-1}$ produziu maior deposição de calda do que a $200 \mathrm{~L} \mathrm{ha}^{-1}$ para ambas as pontas de pulverização e não reduziu a eficácia dos inseticidas. Ambos os inseticidas tiveram eficácias semelhantes no controle da lagarta-do-cartucho aos 10 dias após a aplicação (DAA), embora a eficácia do inseticida fenpropatrina tenha sido maior do que a do triflumuron aos 3 DAA.
\end{abstract}

Palavras-chave: lagarta-do-cartucho, Zea mays, ponta de pulverização, taxa de aplicação, manejo de pragas.

\section{INSECTICIDE APPLICATION TECHNOLOGY ON FALL ARMYWORM CONTROL IN CORN}

\begin{abstract}
The fall armyworm (Spodoptera frugiperda) is a key pest in corn, and insecticide applications have been needed to reduce the insect population. The fall armyworm has been difficult to control because its larvae is commonly protected in the whorl of corn, which may reduce insecticide deposition and its efficacy. Therefore, this research aimed to evaluate the effects of application rate, nozzle type and insecticide on control of $S$. frugiperda in corn. Experiment was conducted in a randomized complete block design and a $2 \times 2 \times 2+1$ factorial scheme with four replications. The factors were two application rates (100 e $\left.200 \mathrm{~L} \mathrm{ha}^{-1}\right)$, two hydraulic nozzles (standard and air induction flat-fan) and two insecticides (triflumuron and fenpropathrin). An additional treatment was also included, composed of chlorpyrifos sprayed at $200 \mathrm{~L} \mathrm{ha}^{-1}$ using a standard flat-fan nozzle. Parameters of interest were droplet spectrum, spray deposition, biological efficacy and crop yield. A $100 \mathrm{~L} \mathrm{ha}^{-1}$ application rate produced greater spray deposition than at $200 \mathrm{~L} \mathrm{ha}^{-1}$ for both nozzle types and did not reduce the insecticide efficacy. At 10 days after application (DAA) both insecticides provided similar efficacy on fall armyworm control, although it was greater using fenpropathrin than triflumuron at 3 DAA. Keywords: fall armyworm, Zea mays, spray nozzle, spray volume, pest management.
\end{abstract}


O milho (Zea mays L.) é o principal cereal produzido no Brasil. No seu cultivo, diversos fatores podem comprometer a produtividade, sendo que segundo Michelotto et al. (2011) a lagarta-do-cartucho, Spodoptera frugiperda (J.E. Smith, 1797) (Lepidoptera: Noctuidae), é um dos fatores mais limitantes, classificada como praga-chave desta cultura. Especialmente na segunda safra, por causa das condições climáticas favoráveis e dos restos culturais, este inseto-praga prejudica ainda mais a produção da cultura, com o surgimento de gerações consecutivas e cada vez mais resistentes aos inseticidas utilizados (Santiago et al., 2008).

Biologicamente, as posturas efetuadas pelos adultos iniciam-se com o surgimento das primeiras folhas, logo após a emergência das plantas de milho. Nesta fase inicial, as lagartas de primeiro instar começam a alimentação raspando a epiderme e o parênquima foliar, caracterizando os primeiros sintomas nas folhas. Essas lesões se tornam mais severas à medida que as lagartas se desenvolvem, podendo causar até a destruição total das plantas mais novas. Dessa forma, há reduções significativas de produtividade, com perdas de até $100 \%$, se não forem adotadas táticas de manejo (Michelotto et al., 2011).

Além do uso de cultivares transgênicas, o uso de inseticidas é uma ferramenta eficaz e constantemente adotada por produtores, visando reduzir a população da praga e, consequentemente, o número de plantas atacadas e seu possível dano à cultura (Tomquelski \& Martins, 2007). Dentre os inseticidas mais utilizados, pode-se citar o grupo químico das benzoilureias, caracterizadas pela sua alta seletividade e atuação como reguladores de crescimento; e piretroides e organofosforados, conhecidos como neurotóxicos de amplo espectro (Toscano et al., 2012), não sendo seletivos a inimigos naturais e a organismos não alvo (Nascimento, 2012).

O controle químico efetivo de $S$. frugiperda tem encontrado inúmeras dificuldades, entre elas, o desenvolvimento de resistência a organofosforados, carbamatos, neonicotinoides e reguladores de crescimento (Yu, 2008), o que leva à constante rotação de produtos com diferentes mecanismos de ação, assim como à aplicação de inseticidas específicos para os diferentes estágios da praga (Castle et al., 2010).

A tecnologia de aplicação, contudo, torna-se um fator de extrema relevância quando se considera a adoção da aplicação de inseticidas, que deverá garantir a deposição do produto no alvo desejado, de forma eficiente, e ainda se evitando perdas para o ambiente (Cunha, 2008; Van Zyl et al., 2013). Nesse sentido, a adoção adequada da taxa de aplicação, das pontas utilizadas, do tamanho e da densidade de gotas mais uniformes são fatores fundamentais para que os inseticidas não percam sua eficácia (Souza et al., 2012).

Para a tecnologia de aplicação, o controle de $S$. frugiperda ainda apresenta mais desafios, pois esta praga se aloja no interior do cartucho do milho, sendo um local de difícil alcance para o ingrediente ativo, podendo comprometer a sua atuação caso o espectro de gotas não seja adequado e a taxa de aplicação não seja ajustada. Por isso, é importante que se use tecnologia que proporcione deposição de gotas mais eficiente, principalmente na parte superior do dossel do milho.

Ferguson et al. (2016) mencionaram que a taxa de aplicação tem efeito sobre a performance dos pesticidas. Maiores taxas podem elevar a quantidade de produtos no alvo até certo ponto, a partir do qual pode ocorrer escorrimento e perdas para o solo, prejudicando o ambiente. Por outro lado, em alguns casos, menores volumes de calda podem aumentar a cobertura e a eficácia dos produtos (Fritz et al., 2007), além de 
aumentar a capacidade operacional de pulverizadores e diminuir custos de produção, como defendido por Farinha et al. (2009).

Desta forma, o objetivo deste trabalho foi avaliar o efeito de diferentes taxas de aplicação e pontas de pulverização na eficácia de inseticidas neurotóxicos e reguladores de crescimento no controle de $S$. frugiperda na cultura do milho.

\section{Material e Métodos}

\section{Delineamento experimental}

O experimento foi conduzido durante o outono de 2015 na Fazenda Experimental Capim Branco, pertencente à Universidade Federal de Uberlândia (UFU), no Município de Uberlândia, Minas Gerais, a uma altitude de 842 metros e coordenadas geográficas de $18^{\circ} 53^{\prime} 23,46^{\prime \prime} \mathrm{S}$ de latitude e $48^{\circ} 20^{\prime} 27,46^{\prime \prime} \mathrm{O}$ de longitude.

Implantou-se o experimento com milho híbrido convencional Dow 2B537, semeado em linhas de cultivo espaçadas por $0,5 \mathrm{~m}$ e densidade populacional de

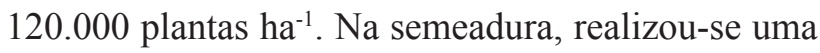
adubação, localizada na linha de cultivo, do formulado NPK 08-28-16 na dose de $280 \mathrm{~kg} \mathrm{ha}^{-1}$, de acordo com a fertilidade pré-amostrada do solo e a exigência da cultura. Realizaram-se duas aplicações de herbicidas na área, aos 5 dias após a semeadura (DAS) em pré-emergência, e aos 20 DAS em pós-emergência. Ao atingir o estádio fenológico V6/V7, a cultura recebeu uma adubação de cobertura do formulado NPK 20-00-20 na dose de $300 \mathrm{~kg} \mathrm{ha}^{-1}$.

O delineamento experimental utilizado foi de blocos casualizados (DBC), com nove tratamentos e quatro repetições, totalizando 36 parcelas, sendo que cada parcela foi constituída de $20 \mathrm{~m}^{2}$, sendo oito linhas de plantas com 5,0 m de comprimento cada. Como parcela útil foram consideradas as quatro linhas centrais, desprezando-se $0,5 \mathrm{~m}$ de cada extremidade. As $2^{\mathrm{a}}$ e $7^{\mathrm{a}}$ linhas foram utilizadas para se obter a produtividade do híbrido ao final do período produtivo.

Os tratamentos foram dispostos em esquema fatorial $2 \times 2 \times 2+1$, sendo fatores as taxas de aplicação de 100 e $200 \mathrm{~L} \mathrm{ha}^{-1}$, as pontas BD (11002 jato plano simples) e ADIA (11002 jato plano com indução de ar) e os produtos Certero ${ }^{\circledR}$ (triflumuron $480 \mathrm{~g}$ $\mathrm{L}^{-1}$, regulador de crescimento) e Danimen $300 \mathrm{CE}^{\circledR}$ (fenpropatrina $300 \mathrm{~g} \mathrm{~L}^{-1}$, piretroide), ambos na dose de $100 \mathrm{~mL}$ do produto comercial ha ${ }^{-1}$. Além disso, optou-se por mais um tratamento controle, com a aplicação de Lorsban 480 BR (clorpirifós $480 \mathrm{~g} \mathrm{~L}^{-1}$, organofosforado) a $200 \mathrm{~L} \mathrm{ha}^{-1}$ com a ponta $\mathrm{BD}$, por ser este o tratamento comumente utilizado na Fazenda Experimental. A Tabela 1 descreve os tratamentos avaliados.

\section{Aplicações e avaliações de eficácia biológica}

Para a aplicação dos tratamentos, utilizou-se um pulverizador costal acionado por pressão constante $\left(\mathrm{CO}_{2}\right)$, com quatro pontas espaçadas entre si por $0,5 \mathrm{~m}$. Adotou-se a pressão de $300 \mathrm{kPa}$ em todos os tratamentos, variando as velocidades de trabalho de acordo com as taxas de aplicação de 100 e $200 \mathrm{~L} \mathrm{ha}^{-1}$ (8 e $4 \mathrm{~km} \mathrm{~h}^{-1}$, respectivamente).

As aplicações dos tratamentos ocorreram aos 23 dias após a emergência da cultura (DAE), quando esta se encontrava em estádio vegetativo V6, e aos 30 DAE repetiram-se os tratamentos na área. Previamente às aplicações, efetuou-se uma avaliação na área do experimento para a caracterização quanto à presença do inseto-alvo. Para tanto, foram amostra- 
Tabela 1. Descrição dos tratamentos avaliados na tecnologia de aplicação de inseticidas no milho.

\begin{tabular}{cccc}
\hline Tratamento & Inseticida & Ponta & Taxa de aplicação $\left(\mathrm{L} \mathrm{ha}^{-1}\right)$ \\
\hline 1 & fenpropatrina & BD11002 & 100 \\
2 & fenpropatrina & BD11002 & 200 \\
3 & fenpropatrina & ADIA 11002 & 100 \\
4 & fenpropatrina & ADIA 11002 & 200 \\
5 & triflumuron & BD11002 & 100 \\
6 & triflumuron & BD1 1002 & 200 \\
7 & triflumuron & ADIA 11002 & 100 \\
8 & triflumuron & ADIA 11002 & 200 \\
9 & clorpirifós & BD11002 & 200 \\
\hline
\end{tabular}

das oito plantas por parcela, das quais se arrancou o cartucho (folhas internas que ainda não estavam completamente abertas) e contou-se o número de lagartas vivas. Além disso, escolheram-se aleatoriamente 25 plantas da área útil da parcela, e atribuíram-lhes notas visuais de 0 a 9 para avaliação de dano, de acordo com a metodologia de Fernandes et al. (2003) e com a escala adaptada de Davis et al. (1992). A avaliação quanto à presença das lagartas foi realizada novamente aos 3, 7, 10 e 14 dias após a $1^{a}$ aplicação (DAA), utilizando a mesma metodologia da avaliação prévia.

\section{Análise do espectro} de gotas e da deposição

Adicionalmente, avaliou-se o espectro de gotas pulverizadas pelas pontas empregadas, através de papéis hidrossensíveis com dimensões de 76 × $26 \mathrm{~mm}$ (Spraying Systems Co., Wheaton, USA). Antes da pulverização, foram colocados dois papéis hidrossensíveis por parcela, em posição horizontal e voltados para cima. Em laboratório, os papéis foram digitalizados com um scanner (resolução espacial de 600 dpi não interpolados, com cores em 24 bits) e analisados em programa computacional CIR 1.5 (Conteo y Ti- pificación de Impactos de Pulverización), específico para essa finalidade. Determinou-se o diâmetro da mediana volumétrica (DMV), a amplitude relativa (AR) e a porcentagem do volume de gotas com diâmetro inferior a $100 \mu \mathrm{m}(\%<100)$.

Para avaliar a deposição de calda nas plantas de milho, adicionou-se à calda de aplicação o marcador Azul Brilhante (Duas Rodas, Jaraguá do Sul, Brasil) na concentração de $2.000 \mathrm{mg} \mathrm{L}^{-1}$, fixo para todos os tratamentos, o qual foi detectado por absorbância em espectrofotometria. De acordo com Pinto et al. (2007), esse corante é estável por um período de até cinco horas de exposição solar. Por causa dessa concentração fixa de marcador na calda, assim como a aplicação de dois volumes de calda diferentes, os dados de deposição foram multiplicados por um fator de correção, conforme Ferreira et al. (2013), baseando-o na maior taxa de aplicação, para, então, permitir a comparação das médias de deposição na análise estatística.

Logo após a aplicação da calda inseticida, coletaram-se ao acaso duas folhas de milho em cada parcela, sendo uma da parte superior e uma da parte inferior das plantas. Como parte superior, escolheu-se a quinta folha totalmente aberta. Já em relação à parte inferior, escolheram-se aquelas posicionadas $15 \mathrm{~cm}$ 
em relação ao solo. Após a coleta, as folhas foram acondicionadas separadamente em sacos plásticos e mantidas em caixa térmica, para posterior manipulação em laboratório.

As condições ambientais durante as aplicações foram monitoradas por meio de um termo-higro-anemômetro digital (Kestrel® 4000, Minneapolis, MN, USA). A temperatura durante as aplicações variou de 23,0 a $28,6{ }^{\circ} \mathrm{C}$; a umidade relativa variou de 64,6 a $75,6 \%$; e a velocidade do vento, entre 1,6 e $4,2 \mathrm{~km} \mathrm{~h}^{-1}$.

As análises laboratoriais de deposição foram feitas no Laboratório de Mecanização Agrícola (LAMEC), pertencente à UFU. Para isso, foram adicionados $70 \mathrm{~mL}$ de água destilada aos sacos plásticos contendo as folhas superiores e inferiores. Os sacos foram, então, lavados e agitados por 30 segundos, para máxima extração possível do marcador presente nas amostras. Em seguida, o líquido foi retirado e depositado em copos plásticos, os quais foram acondicionados em local refrigerado provido de isolamento luminoso por 24 horas, para posterior leitura de absorbância no espectrofotômetro.

Utilizou-se um espectrofotômetro com lâmpada de tungstênio-halogênio (Biospectro, SP22, Curitiba-PR, Brasil) para realizar as leituras. A quantificação da coloração foi feita por absorbância em 630 nm. Com o uso de curvas de calibração, obtidas por meio de soluções-padrão do marcador, os dados de absorbância foram transformados em concentração $\left(\mathrm{mg} \mathrm{L}^{-1}\right)$. De posse da concentração inicial da calda e do volume de diluição das amostras, determinou-se a massa do marcador retida nas folhas de milho coletadas nas parcelas, corrigindo o valor de acordo com a taxa aplicada. O depósito total foi dividido pela massa seca de cada amostra, obtendo-se assim a quantidade em micrograma $(\mu \mathrm{g})$ de marcador por grama $(\mathrm{g})$ de massa seca. As folhas foram secas em estufa de circulação forçada, a $65^{\circ} \mathrm{C}$, por 72 horas.

\section{Avaliação de produtividade}

Avaliou-se a produtividade nas parcelas experimentais, colhendo-se todas as espigas das $2^{\mathrm{a}}$ e $7^{\mathrm{a}}$ linhas de cultivo no comprimento de $4 \mathrm{~m}$, quando a cultura se encontrava no estádio reprodutivo R6 (maturidade fisiológica). Desprezou-se 0,5 m de cada extremidade da parcela. Em seguida, efetuou-se a debulha através de uma debulhadora elétrica, pesaram-se os grãos em balança analítica, corrigiu-se a umidade para $14 \%$ e extrapolou-se a massa obtida para a produtividade por hectare $\left(\mathrm{kg} \mathrm{ha}^{-1}\right)$. Por fim, conforme metodologia do Ministério da Agricultura, Pecuária e Abastecimento (Brasil, 2009), constatou-se também a massa (g) de 1.000 grãos de milho.

\section{Análises estatísticas}

Os dados de deposição e eficácia de controle dos tratamentos foram primeiramente submetidos aos testes de normalidade de distribuição dos resíduos, homogeneidade das variâncias e aditividade de blocos pelos testes de Shapiro Wilk, Levene e Tukey, respectivamente, a 0,01 de significância, utilizando o programa SPSS Inc., Chicago, IL, USA. Nos casos em que as pressuposições não foram atendidas, os dados foram transformados em $\sqrt{x}$ e submetidos à nova análise. Somente quando a transformação corrigiu ou pelo menos melhorou uma das pressuposições sem prejudicar as demais, utilizaram-se os dados transformados para a análise de variância. Do contrário, utilizaram-se os dados originais.

Quando pertinente, procedeu-se o teste de F por meio da análise de variância, a comparação das 
médias pelo teste de Tukey e um contraste com o tratamento controle pelo teste de Dunnett, ambos a 0,05 de significância, com o programa estatístico ASSISTAT 7.7 beta (Silva, 2013). As características do espectro de gotas das pontas BD e ADIA foram comparadas por meio da análise do teste $t$ de Student para amostras independentes, a 0,05 de significância.

\section{Resultados e Discussão}

A partir da análise do espectro de gotas (Tabela 2), verificou-se que a ponta de jato plano convencional (BD) produziu gotas com menores diâmetros (DMV) e menor uniformidade, em razão da maior amplitude relativa (AR). Consequentemente, houve maior quantidade de gotas sujeitas à deriva $(\%<100$ $\mu \mathrm{m})$. As pontas BD e ADIA originaram gotas com DMV de 267 e $499 \mu \mathrm{m}$, respectivamente, classificadas como gotas médias e muito grossas de acordo com a Norma ASAE S-572.1 (American Society of Agricultural and Biological Engineers, 2009). Em relação ao risco de deriva $(\%<100 \mu \mathrm{m})$, a ponta ADIA gerou valores menores, no entanto, ambas as pontas geraram baixa quantidade de gotas propensas à deriva. Sobre isso, Cunha et al. (2003) afirmam que valores abaixo de $15 \%$ de volume de gotas com diâmetro inferior a $100 \mu \mathrm{m}$ podem implicar pulverizações mais seguras.

Os resultados de deposição de calda, na região inferior da cultura, não apresentaram diferença significativa entre os tratamentos, com valor médio de $341,15 \mu \mathrm{g} \mathrm{g}^{-1}$. Porém, em relação à parte superior da cultura, os resultados apresentaram interação significativa entre tipo de ponta e taxa de aplicação (Tabela 3 ), sendo que a menor taxa com $100 \mathrm{~L} \mathrm{ha}^{-1}$ proporcionou maior quantidade de calda depositada, com ambas as pontas de pulverização.
Esses resultados concordam com Fritz et al. (2007) quanto à viabilidade na redução da taxa de aplicação, a fim de garantir maior deposição no dossel superior da cultura, e consequentemente, menores barreiras na atuação dos inseticidas e na eficácia no controle de $S$. frugiperda. Além disso, uma das grandes vantagens de se usar menores taxas de aplicação é a redução de perdas para o ambiente, principalmente por escorrimento.

Resultados semelhantes foram obtidos por Campos et al. (2011), que, ao estudarem a ponta de pré-orifício DG e a ponta com indução de ar AI, com as taxas de 50, 100 e $150 \mathrm{~L} \mathrm{ha}^{-1}$, também verificaram a possibilidade de redução das taxas de aplicação, sem afetar a qualidade do tratamento. Por outro lado, Cunha e Silva Júnior (2010), também verificando a influência de diferentes tecnologias de aplicação para controle de S. frugiperda na cultura do sorgo, observaram que a taxa de $130 \mathrm{~L} \mathrm{ha}^{-1}$ em comparação a $200 \mathrm{~L} \mathrm{ha}^{-1}$, usando a ponta de jato plano defletor com indução de ar, apresentou menor deposição na parte superior. Tais divergências possivelmente se devem às diferentes estruturas dos cultivos avaliados.

Em relação à eficácia biológica dos inseticidas no controle de $S$. frugiperda, nas avaliações prévias antes das aplicações, constatou-se na área experimental uma média de 2,78 lagartas por planta de milho, com nota 6,63 na escala de danos, e com uniformidade de distribuição do inseto-praga. Na primeira avaliação após as aplicações, aos 3 dias, houve uma interação tripla entre os fatores (volume de calda, ponta de pulverização e inseticidas), conforme Tabela 4. É possível observar que o inseticida triflumuron apresentou maior número médio de lagartas em relação ao fenpropatrina com a ponta de pulverização $\mathrm{BD}$ e taxa de aplicação de $100 \mathrm{~L} \mathrm{ha}^{-1}$. 
Tabela 2. Diâmetro da mediana volumétrica (DMV, $\mu \mathrm{m})$, amplitude relativa (AR) e porcentagem do volume composto por gotas com diâmetro inferior a $100 \mu \mathrm{m}(\%<100, \%)$ em aplicação com pontas de jato plano (BD) e jato plano com indução de ar (ADIA) de inseticidas na cultura do milho.

\begin{tabular}{cccc}
\hline Ponta & DMV $(\mu \mathrm{m})$ & AR & $\%<100 \mu \mathrm{m}(\%)$ \\
BD & $267,00 \mathrm{~b}$ & $1,25 \mathrm{a}$ & $2,61 \mathrm{a}$ \\
ADIA & $498,92 \mathrm{a}$ & $1,08 \mathrm{~b}$ & $1,11 \mathrm{~b}$ \\
\hline $\mathrm{t}$ & $3,75 \mathrm{E}-8^{* *}$ & $5,31 \mathrm{E}-3^{* *}$ & $1,60 \mathrm{E}-8^{* *}$ \\
C.V. $(\%)$ & 20,08 & 13,48 & 18,57 \\
\hline
\end{tabular}

Médias seguidas por letras distintas nas colunas diferem entre si pelo teste de t de Student, a 0,05. t: valor de t calculado. C.V.: coeficiente de variação. **significativo a 0,01 .

Tabela 3. Deposição de calda $\left(\mu \mathrm{g} \mathrm{g}^{-1}\right)$ na parte superior de plantas de milho após a aplicação de inseticidas com diferentes tecnologias de aplicação.

\begin{tabular}{|c|c|c|}
\hline \multirow{2}{*}{ Ponta } & \multicolumn{2}{|c|}{ Taxa de aplicação $\left(\mathrm{L} \mathrm{ha}^{-1}\right)$} \\
\hline & 100 & 200 \\
\hline $\mathrm{BD}$ & $243,39 \mathrm{aA}$ & $184,66 \mathrm{aB}$ \\
\hline ADIA & $200,84 \mathrm{aA}$ & $148,02 \mathrm{aB}$ \\
\hline \multicolumn{2}{|c|}{ Controle } & \\
\hline \multicolumn{3}{|c|}{$\begin{array}{c}\mathrm{Fc}_{\text {int }}=0,0007 * ; \mathrm{Fc}_{\text {Dunnett }}=0,230^{\text {ns }} ; \mathrm{C} . \mathrm{V} .=11,54 \% \\
\mathrm{~F}_{\text {Levene }}=\mathbf{2 , 6 6 6} ; \mathrm{W}=\mathbf{0 , 9 6 6} ; \mathrm{F}_{\text {Tukey }}=\mathbf{0 , 5 8 3}\end{array}$} \\
\hline
\end{tabular}

Médias seguidas por letras iguais, minúsculas nas colunas e maiúsculas nas linhas, não diferem entre si pelo teste de Tukey, a 0,05 . $\mathrm{Fc}_{\text {int }}$ : valor de $\mathrm{F}$ calculado para a interação entre ponta e taxa de aplicação. $\mathrm{Fc}_{\text {Dunnet }}$ : valor de $\mathrm{F}$ calculado para a interação entre o fatorial e o tratamento adicional. C.V.: coeficiente de variação. Valores em negrito representam variâncias homogêneas $\left(\mathrm{F}_{\text {Levene }}\right)$, resíduos com distribuição normal (W) e blocos com efeitos aditivos $\left(\mathrm{F}_{\text {Tukey }}\right)$, pelos testes de Levene, Shapiro-Wilk e Tukey, respectivamente. ${ }^{\times}$dados transformados por $\sqrt{\mathrm{x}}$; * significativo a 0,05 . ${ }^{\text {ns }}$ não significativo.

De acordo com IRAC-BR (2015), a diferença entre os produtos no controle populacional da lagarta pode ser explicada pelo modo de ação dos inseticidas, sendo que o triflumuron (inibidor da síntese de quitina) é um produto recomendado para início de infestação das lagartas no milho, apresentando ação relativamente mais lenta que os neurotóxicos fenpropatrina (modulador dos canais de sódio) e clorpirifós (inibidor da acetilcolinesterase), os quais apresentam ação mais rápida. Já aos 10 DAA, não houve diferença entre o número médio de lagartas entres os ingredientes ativos.

Apesar de terem ocorrido poucas diferenças entre as tecnologias de aplicação quanto à eficácia de controle da $S$. frugiperda, é importante ressaltar que a menor taxa de aplicação (100 L ha-1) não apresentou desempenho inferior à taxa de $200 \mathrm{~L} \mathrm{ha}^{-1}$, situação também observada entre as pontas de pulverização. Esses resultados relacionados às maiores deposições de calda reforçam a opção pelos tratamentos com a ponta ADIA (jato plano com indução de ar) e a taxa de $100 \mathrm{~L} \mathrm{ha}^{-1}$, sem comprometer a eficiência de controle.

Campos et al. (2011), de maneira semelhante ao presente trabalho, também observou controle populacional equiparado com menores volumes de calda, possibilitando, em seu caso, reduzir de 150 para até $50 \mathrm{~L} \mathrm{ha}^{-1}$ em aplicações para controle de $S$. frugi- 
Tabela 4. Número médio de lagartas vivas de S. frugiperda por planta de milho aos 3 e 10 dias após a aplicação (DAA) de fenpropatrina, triflumuron e clorpirifós, utilizando-se diferentes pontas e taxas de aplicação.

\begin{tabular}{|c|c|c|c|}
\hline \multicolumn{4}{|c|}{3 DAA } \\
\hline \multirow{2}{*}{ Inseticida } & \multirow{2}{*}{ Ponta } & \multicolumn{2}{|c|}{ Taxa de aplicação $\left(\mathrm{L} \mathrm{ha}^{-1}\right)$} \\
\hline & & 100 & 200 \\
\hline \multirow{2}{*}{ fenpropatrina } & $\mathrm{BD}$ & $1,37 \mathrm{aA}$ & $1,03 \mathrm{aA}$ \\
\hline & ADIA & $1,12 \mathrm{aA}$ & $1,16 \mathrm{aA}$ \\
\hline \multirow{2}{*}{ triflumuron } & $\mathrm{BD}$ & $2,25 \mathrm{bA}$ & $1,78 \mathrm{aA}$ \\
\hline & ADIA & $1,65 \mathrm{abA}$ & $1,56 \mathrm{aA}$ \\
\hline \multirow{2}{*}{ Ponta } & Taxa de aplicação & \multicolumn{2}{|c|}{ Inseticida } \\
\hline & $\left(\mathrm{L} \mathrm{ha}^{-1}\right)$ & fenpropatrina & triflumuron \\
\hline \multirow{2}{*}{$\mathrm{BD}$} & 100 & $1,37 \mathrm{~A}$ & $2,25 \mathrm{~B}$ \\
\hline & 200 & $1,03 \mathrm{~A}$ & $1,78 \mathrm{~B}$ \\
\hline \multirow{2}{*}{ ADIA } & 100 & $1,12 \mathrm{~A}$ & $1,65 \mathrm{~A}$ \\
\hline & 200 & $1,16 \mathrm{~A}$ & $1,56 \mathrm{~A}$ \\
\hline \multicolumn{2}{|c|}{ Controle $^{\text {ns }}$} & \multicolumn{2}{|c|}{1,94} \\
\hline \multicolumn{4}{|c|}{$\begin{array}{c}\mathrm{Fc}=0,000^{* * *} ; \mathrm{Fc}_{\text {Dunnett }}=3,727^{\mathrm{ns}} ; \mathrm{C} . \mathrm{V} .=28,21 \% \\
\mathrm{~F}_{\text {Levene }}=\mathbf{1}, \mathbf{4 5 6} ; \mathrm{W}=\mathbf{0 , 9 6 2} ; \mathrm{F}_{\text {Tukey }}=\mathbf{0 , 0 0 2}\end{array}$} \\
\hline \multicolumn{4}{|c|}{10 DAA } \\
\hline \multirow{2}{*}{ Produto } & \multirow{2}{*}{ Ponta } & \multicolumn{2}{|c|}{ Taxa de aplicação $\left(\mathrm{L} \mathrm{ha}^{-1}\right)$} \\
\hline & & 100 & 200 \\
\hline \multirow{2}{*}{ fenpropatrina } & $\mathrm{BD}$ & $0,50 \mathrm{aA}$ & $0,72 \mathrm{aA}$ \\
\hline & ADIA & $0,44 \mathrm{aA}$ & $0,31 \mathrm{aA}$ \\
\hline \multirow{2}{*}{ triflumuron } & $\mathrm{BD}$ & $1,03 \mathrm{aA}$ & $0,59 \mathrm{aA}$ \\
\hline & ADIA & $0,72 \mathrm{aA}$ & $0,56 \mathrm{aA}$ \\
\hline \multicolumn{2}{|c|}{ Controle $^{+}$} & \multicolumn{2}{|c|}{1,41} \\
\hline \multicolumn{4}{|c|}{$\begin{array}{c}\mathrm{Fc}_{\text {int }}=0,899^{\mathrm{ns}} ; \mathrm{Fc}_{\text {Dunnett }}=10,390^{* *} ; \mathrm{C} . \mathrm{V} .=66,79 \% \\
\mathrm{~F}_{\text {Levene }}=\mathbf{1 , 5 0 9} ; \mathrm{W}=\mathbf{0 , 9 8 2} ; \mathrm{F}_{\text {Tukey }}=\mathbf{0 , 5 8 6}\end{array}$} \\
\hline
\end{tabular}

Médias seguidas por letras iguais, minúsculas nas colunas e maiúsculas nas linhas, não diferem entre si pelo teste de Tukey, a 0,05 . $\mathrm{Fc}$ : valor de $\mathrm{F}$ calculado para a interação tripla. $\mathrm{Fc}_{\text {Dunnet }}$ : valor de $\mathrm{F}$ calculado para a interação entre o fatorial e o tratamento adicional. C.V.: coeficiente de variação. Valores em negrito representam variâncias homogêneas $\left(\mathrm{F}_{\text {Levene }}\right)$, resíduos com distribuição normal (W) e blocos com efeitos aditivos $\left(\mathrm{F}_{\text {Tukey }}\right)$, pelos testes de Levene, Shapiro-Wilk e Tukey, respectivamente. ** significativo a $0,01 .{ }^{+}$todos os tratamentos diferiram do controle. ${ }^{\text {ns }}$ não significativo.

perda. No entanto, Gimenes et al. (2012), ao avaliarem o controle da mesma praga com as taxas de $100 \mathrm{~L}$ ha-1 e $200 \mathrm{~L} \mathrm{ha}^{-1}$ em aplicação em área total, verificaram melhor controle com a maior taxa em virtude da maior cobertura de gotas. Para Campos et al. (2011), a taxa reduzida apresentou desempenho satisfatório por causa do uso de pontas de pulverização redutoras de deriva, ou seja, menores taxas de aplicação combi- nadas com gotas de maior diâmetro, menos propensas à deriva, podem proporcionar um controle fitossanitário mais sustentável.

Em geral, aos 7, 10 e 14 DAA não houve diferença entre o número médio de lagartas, pois se tratam de intervalos entre aplicações, nos quais a ação dos produtos já está em processo de redução. Apesar do triflumuron ter apresentado controle populacional 
inferior ao fenpropatrina aos 3 DAA, não houve diferença em relação ao clorpirifós neste período. No entanto, ao avançar no período pós-tratamento, aos 10 DAA, o controle populacional da praga pelo inseticida regulador de crescimento e o piretroide superaram o controle pelo organofosforado. Isto mostra a viabilidade em se optar pelos dois primeiros produtos em relação ao último. É importante ressaltar que, diante do manejo integrado de pragas e da conservação de insetos e organismos benéficos, o triflumuron é preferível, pois apresenta maior seletividade, se comparado aos outros dois, que são de amplo espectro de ação e com menor seletividade.

Para Corrêa et al. (2012), a utilização de inseticidas reguladores de crescimento é recomendada, pois a seletividade é a chave do manejo de pragas em sistemas que visam reduzir a população de insetos nocivos, afetando o mínimo possível outros componentes do agroecossistema. Estes autores, ao avaliarem o efeito da aplicação do lufenuron, um regulador de crescimento do mesmo grupo que o triflumuron, sobre o controle da lagarta-do-cartucho na cultura do milho, verificaram controle satisfatório por parte deste inseticida. No entanto, não houve aumento de produtividade em comparação com os outros tratamentos.

As notas na escala de danos provocados pelo ataque da lagarta-do-cartucho no milho estão apresentadas na Tabela 5. Não houve interação tripla entre os fatores em nenhuma das avaliações, mas houve interação entre os produtos e as pontas aos 3 e 10 DAA, e interação entre os produtos e as taxas aos 7 DAA. Aos 14 DAA, houve diferença apenas em relação ao tratamento adicional.

Em concordância com os resultados de número de lagartas por planta de milho (Tabela 4), as notas de danos também mostraram que, aos 3 DAA, as plan- tas que receberam a aplicação de triflumuron tiveram maiores danos em relação à aplicação de fenpropatrina com a ponta ADIA, mas isso não se refletiu no decorrer das avaliações e, em geral, ambos foram melhores que o clorpirifós. É importante levantar a possibilidade de resistência da S. frugiperda ao ingrediente ativo clorpirifós, já que este é usado diversas vezes na mesma safra como padrão de controle deste inseto-praga na região do experimento. Além disso, as infestações da lagarta são recorrentes e cada vez mais severas, fato também constatado por Tomquelski e Martins (2007).

Em relação à eficiência das tecnologias de aplicação, novamente é reforçada a possibilidade de adoção de taxas reduzidas de aplicação e pontas com gotas mais grossas. A exceção foi aos 10 DAA, na qual as aplicações de triflumuron com a ponta ADIA geraram maiores notas na escala de dano, porém isto não se refletiu na quantidade de lagartas, como visto na Tabela 4, mostrando a eficiência equiparada entre as duas pontas.

Em relação à produtividade final das parcelas experimentais e à massa de 1.000 grãos, não houve diferença entre as tecnologias empregadas. Como médias gerais, foram obtidos $5.759,81 \mathrm{~kg} \mathrm{ha}^{-1} \mathrm{e}$ 248,40 g, respectivamente. Apesar de Cunha e Silva Júnior (2010) explicarem que há relação positiva entre eficiência de controle e produtividade, isto não foi observado no presente trabalho, já que mesmo diante dos melhores resultados obtidos por triflumuron e fenpropatrina em relação ao clorpirifós, isto não se refletiu na produtividade final. Por outro lado, assim como observado neste trabalho, Campos et al. (2011) também não verificou diferenças na produtividade final, sendo que outras condições, bem como a intensidade do ataque da lagarta, podem existir no campo e interferir no desempenho da cultura. 
Tabela 5. Notas na escala de danos provocados por lagartas de $S$. frugiperda em plantas de milho aos 3, 7, 10 e 14 dias após a aplicação (DAA) de fenpropatrina, triflumuron e clorpirifós, utilizando-se diferentes pontas e taxas de aplicação.

\section{DAA}

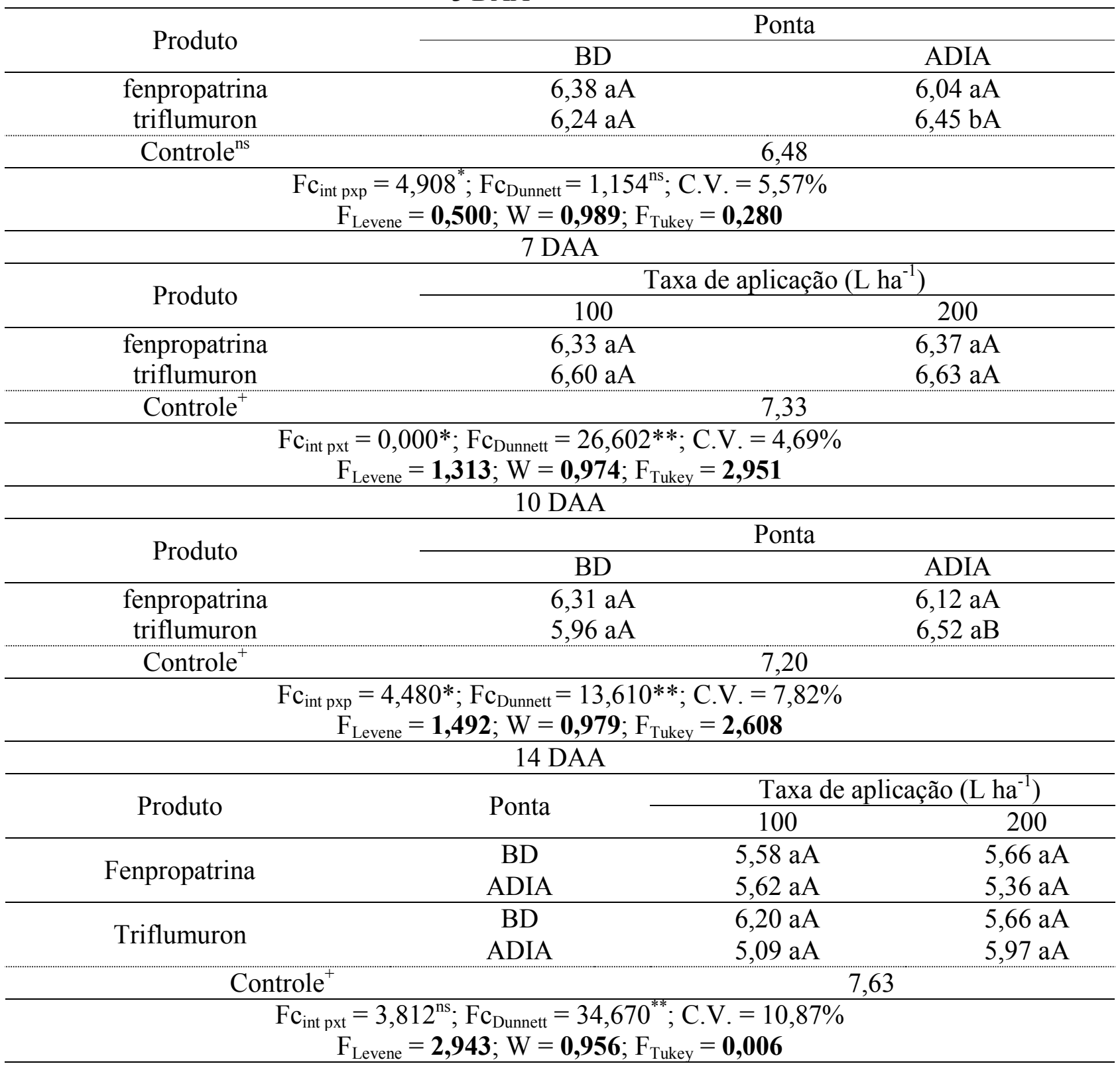

Médias seguidas por letras iguais, minúsculas nas colunas e maiúsculas nas linhas, não diferem entre si pelo teste de Tukey, a 0,05 . $\mathrm{Fc}_{\text {int pxp }}$ : valor de $\mathrm{F}$ calculado para a interação entre ponta e produto. $\mathrm{Fc}_{\text {int pxt }}$ : valor de $\mathrm{F}$ calculado para a interação entre ponta e taxa de aplicação. $\mathrm{Fc}_{\text {Dunnet }}$ : valor de $\mathrm{F}$ calculado para a interação entre o fatorial e o tratamento adicional. C.V.: coeficiente de variação. Valores em negrito representam variâncias homogêneas $\left(\mathrm{F}_{\text {Levene }}\right)$, resíduos com distribuição normal $(\mathrm{W})$ e blocos com efeitos aditivos $\left(\mathrm{F}_{\text {Tukey }}\right)$, pelos testes de Levene, Shapiro-Wilk e Tukey, respectivamente. **significativo a 0,01; *significativo a 0,05 . ${ }^{+}$todos os tratamentos diferiram do controle. ${ }^{\text {ns }}$ não significativo. 


\section{Conclusões}

Menores taxas de aplicação permitem maior deposição e não comprometem a eficácia dos inseticidas, demonstrando a viabilidade técnica desta operação para reduzir custos e perdas.

A ponta de jato plano com indução de ar pode aumentar a segurança ambiental durante a aplicação de inseticidas, mantendo a eficácia do tratamento.

$\mathrm{O}$ inseticida piretroide apresenta eficácia de controle de forma mais rápida que o regulador de crescimento, mas ambos proporcionam semelhante eficácia aos 10 e 14 dias após a aplicação para S. frugiperda.

\section{Agradecimentos}

Os autores agradecem ao CNPq e à Fapemig pela concessão de auxílio financeiro.

\section{Referências}

\section{AMERICAN SOCIETY OF AGRICULTURAL} AND BIOLOGICAL ENGINEERS. Spray Nozzle Classification by Droplet Spectra, ANSI/ASAE S572.1. St. Joseph, 2009. 4 p.

BRASIL. Ministério da Agricultura, Pecuária e Abastecimento. Secretaria de Defesa Agropecuária. Regras para ánalise de sementes. Brasília, DF, 2009. $395 \mathrm{p}$.

CAMPOS, H. B. N.; COSTA, L. L.; LASMAR, O.; LEMOS, R. E.; DECARO JÚNIOR, S. T.; FERREIRA, M. C. Aspectos gerais e atuais da tecnologia de aplicação de produtos fitossanitários. In: BUSOLI, A. C.; FRAGA, D. F.; SANTOS, L. da C. dos; AGUIAR, J. R. de C. C. de; GRIGOLLI, J. F. J.; JANINI, J. C.; SOUZA, L. A. de; VIANA, M. A.; FUNICHELlI, M. (Ed.). Tópicos em entomologia agrícola. Jaboticabal: Gráfica Multipress, 2011. v. 4, p. 205-218.
CASTLE, J. S.; PALUMBO, J. C.; PRABHAKER, N.; HOROWITZ, A. R.; DENHOLM, I. Ecological determinates of Bemisia tabaci resistance to insecticides. In: PHILIP, A. S.; NARANJO, S. E. Bionomics and management of global pest. New York: Springer Science Bussiness, 2010. p. 423-459.

CORRÊA, M. B.; OLIVEIRA, N. C.; VALICENTE, F. H. Manejo da lagarta-do-cartucho na cultura do milho: aplicação de Baculovirus spodoptera isolado e associado com inseticida. Revista Campo Digital, Campo Mourão, v. 7, n. 1, p. 59-67, 2012.

CUNHA, J. P. A. R.; SILVA JÚNIOR, A. D. Volumes de calda e pontas de pulverização no controle químico de Spodoptera frugiperda na cultura do sorgo forrageiro. Engenharia Agrícola, Jaboticabal, v. 30, n. 4, p. 692-699, 2010.

DOI: 10.1590/S0100-69162010000400013.

CUNHA, J. P. A. R.; TEIXEIRA, M. M.; COURY, J. R.; FERREIRA, L. R. Avaliação de estratégias para a redução da deriva de agrotóxicos em pulverizações hidráulicas. Planta Daninha, Rio de Janeiro, v. 21, n. 10, p. 325-332, 2003.

DOI: $10.1590 / \mathrm{S} 0100-83582003000200019$.

CUNHA, J. P. A. R. Simulação da deriva de agrotóxicos em diferentes condições de pulverização. Ciência e Agrotecnologia, Lavras, v. 32, n. 5, p. 1616-1621, 2008. DOI: $10.1590 / \mathrm{S} 1413-70542008000500039$.

DAVIS, F. M.; NG, S. S.; WILLIAMS, W. P. Visual rating scales for screening whorl-stage corn for resistance to fall armyworm. Mississippi: Agricultural and Forest Experiment Station, 1992. 9 p. (Technical Bulletin, 186). FARINHA，J. V.; MARTINS， D.; COSTA， N. V.; DOMINGOS, V. D. Deposição da calda de pulverização em cultivares de soja no estádio R1. Ciência Rural, Santa Maria, v. 39, n. 6, p. 1738-1744, 2009.

DOI: $10.1590 / \mathrm{S} 0103-84782009000600016$

FERGUSON, J. C.; CHECHETTO, R. G.; HEWITT, A. J.; CHAUHAN, B. S.; ADKINS, S. T.; KRUGER, G. R.; O'DONNELL, C. C. Assessing the deposition and canopy 
penetration of nozzles with different spray qualities in an oat (Avena sativa L.) canopy. Crop Protection, Guildford, v. 81, n. 1, p. 14-19, 2016.

DOI: $10.1016 /$ j.cropro.2015.11.013.

FERNANDES, O. D.; PARRA, J. R. P.; NETO, A. F.; PÍCOLI, R.; BORGATTO, A. F.; CLARICE, G. B.; DEMÉTRIO, C. G. B. Efeito do milho geneticamente modificado Mon810 sobre a lagarta-do-cartucho Spodoptera frugiperda (J. E. Smith, 1797) (Lepidoptera: Noctuidae). Revista Brasileira de Milho e Sorgo, Sete Lagoas, v. 2, n. 2, p. 25-35, 2003.

DOI: $10.18512 / 1980-6477 /$ rbms.v2n2p25-35.

FERREIRA, M. C.; LEITE, G. J.; LASMAR, O. Cobertura e depósito de calda fitossanitária em plantas de café pulverizadas com equipamento original e adaptado para plantas altas. Bioscience Journal, Uberlândia, v. 29, n. 1, p. 1539-1548, 2013.

FRITZ, B. K.; HOFFMANN, W. C.; MARTIN, D. E.; THOMSON, S. J. Aerial application methods for increasing spray deposition on wheat heads. Applied Engineering Agriculture, College Station, v. 23, n. 6, p. 709-715, 2007.

GIMENES, M. J.; RAETANO, C. G.; DAL POGETTO, M. H. F.; PRADO, E. P.; CHRISTOVAM, R. S.; REZENDE, D. T.; COSTA, S. I. A. Air-assistance in spray booms which have different spray volumes and nozzle types for chemically controlling Spodoptera frugiperda on corn. Journal of Plant Protection Research, Poznan, v. 52, n. 2, p. 247-253, 2012.

DOI: 10.2478/v10045-012-0039-y.

IRAC-BR. Comitê Brasileiro de Ação e Resistência a Inseticidas. Modo de ação de inseticidas e acaricidas. Mogi Mirim, 2015. Disponível em: <http://www.irac-br. org/\#!modo-de-ao-de-inseticidas-e-acaricidas/c10s0>. Acesso em: 16 jul. 2015.

MICHELOTTO, M. D.; FINOTO, E. L.; MARTINS, A. L. M.; DUARTE A. P. Interação entre transgênicos (Bt) e inseticidas no controle de pragas-chave em híbridos de milho-safrinha. Arquivos do Instituto Biológico, São Paulo, v. 78, n. 1, p. 71-79, 2011.
NASCIMENTO, A. P. C. Depósito de calda sobre linhagem de milho aplicada por pulverização terrestre e por quimigação para o controle de Spodoptera frugiperda (Smith, 1797) (Lepidoptera: Noctuidade). 2012. 35 f. Dissertação (Mestrado em Fitotecnia) Universidade Federal de Uberlândia, Uberlândia, 2012.

PINTO, J. R.; LOECK, A. E.; SOUZA, R. T. de; LOUZADA, R. S. Estabilidade à exposição solar dos traçantes azul brilhante e amarelo tartrasina utilizados em estudos de deposição de pulverização. Revista Brasileira de Agrosciência, v. 13, n. 1, p. 105-107, 2007.

SANTIAGO, G. P.; PÁDUA, L. E. M.; SILVA, P. R. R.; CARVALHO, E. M. S.; MAIA, C. B. Efeitos de extratos de plantas na biologia de Spodoptera frugiperda (J.E. Smith, 1797) (Lepidoptera: Noctuidae) mantida em dieta artificial. Ciência e Agrotecnologia, Lavras, v. 32, n. 3, p. 792-796, 2008.

DOI: $10.1590 / \mathrm{S} 1413-70542008000300013$.

SILVA, F. A. S. Assistat 7.7 beta. Campina Grande: UFCG, 2013.

SOUZA, L. A.; CUNHA, J. P. A. R.; PAVANIN, L. A. Deposição do herbicida 2,4-D Amina com diferentes volumes e pontas de pulverização em plantas infestantes. Revista Ciência Agronômica, Fortaleza, v. 43, n. 1, p. 7885, 2012. DOI: 10.1590/S1806-66902012000100010.

TOMQUELSKI, G. V.; MARTINS, G. L. M. Eficiência de inseticidas sobre Spodoptera frugiperda (J.E. SMITH, 1797) (Lepidoptera: Noctuidae) em milho na região dos Chapadões. Revista Brasileira de Milho e Sorgo, Sete Lagoas, v. 6, n. 1, p. 26-39, 2007.

DOI: 10.18512/1980-6477/rbms.v6n1p26-39.

TOSCANO, L. C.; CALADO FILHO, G. C.; CARDOSO, A. M.; MARUYAMA, W. I.; TOMQUELSKI, G. V. Impacto de inseticidas sobre Spodoptera frugiperda (Lepidoptera, Noctuidae) e seus inimigos naturais em milho safrinha cultivado em Cassilândia e Chapadão do Sul, MS. Arquivos do Instituto Biológico, São Paulo, v. 79, n. 2, p. 223-231, 2012.

DOI: $10.1590 / \mathrm{S} 1808-16572012000200010$. 
VAN ZYL, J. G.; FOURIE, P. H.; SCHUTTE, G. C. Spray deposition assessment and benchmarks for control of Alternaria brown spot on mandarin leaves with copper oxychloride. Crop Protection, Madison, v. 46, n. 4, p. 8087, 2013. DOI: 10.1016/j.cropro.2012.12.005.
YU, S. The toxicology and biochemistry of insecticides. Boca Raton: CRC Press, 2008. 276 p. 